\title{
A Novel Frechet-Type Probability Distribution: Its Properties and Applications
}

\author{
Muhammad Ali, ${ }^{1}$ Alamgir Khalil, ${ }^{1}$ Wali Khan Mashwani ${ }^{(D},{ }^{2}$ Sharifah Alrajhi, ${ }^{3}$ \\ Sanaa Al-Marzouki, ${ }^{3}$ and Kamal Shah $\mathbb{i D}^{4}$ \\ ${ }^{1}$ Department of Statistics, University of Peshawar, Khyber Pakhtunkhwa, Pakistan \\ ${ }^{2}$ Institute of Numerical Sciences, Kohat University of Science and Technology, Pakistan \\ ${ }^{3}$ Statistics Department, Faculty of Science, King AbdulAziz University, Jeddah, Saudi Arabia \\ ${ }^{4}$ Department of Mathematics, University of Malakand, KPK, Pakistan \\ Correspondence should be addressed to Wali Khan Mashwani; mashwanigr8@gmail.com
}

Received 18 September 2021; Revised 16 November 2021; Accepted 28 December 2021; Published 10 January 2022

Academic Editor: Firdous Khan

Copyright (c) 2022 Muhammad Ali et al. This is an open access article distributed under the Creative Commons Attribution License, which permits unrestricted use, distribution, and reproduction in any medium, provided the original work is properly cited.

\begin{abstract}
In this article, a new lifetime model, referred to as modified Frechet-Rayleigh distribution (MFRD), is developed by accommodating an additional parameter in Rayleigh distribution on the basis of the modified Frechet method. Numerous statistical properties of the suggested model are derived and discussed. The technique of maximum likelihood (ML) estimation is adopted to get estimates of the parameters. The suggested model is very flexible and has the capability to model datasets having both monotonic and nonmonotonic failure rates. The proposed model is applied on two real datasets for checking its performance in comparison with available well-known models. The suggested model has shown outclass performance in comparison with the available versions of the Rayleigh distribution used in the literature.
\end{abstract}

\section{Introduction}

The distribution has many applications. It can be defined as follows.

Let $X \sim N\left(0, \delta^{2}\right)$ and $Y \sim N\left(0, \delta^{2}\right)$ be independent normally distributed random variables. Then, $Z=$ square root of $\left(X^{2}+Y^{2}\right)$ has a Rayleigh distribution.

Rayleigh distribution is widely used in various fields, such as life sciences, agriculture, and biological sciences, and in the analysis of metrological characteristics, such as wind speed. The Rayleigh distribution has applications in situations where sizes of normally distributed variables are vital. Rayleigh distribution is also used in the investigation of wind velocity into its orthogonal XY coordinates. Furthermore, assuming that these components are independently and normally distributed with equal variances and means equal to zero. Thus, the variable of interest is wind speed and can be modeled by a Rayleigh distribution. According to Fernandez et al. [1] and Akhter et al. [2], it has useful applications in the field of medical sciences. In the field of engineering, it is used to find the expected lifespan of material objects, reliability investigation, service periods, and theory of communication. The density function (PDF) and distribution function $(\mathrm{CDF})$ of $\mathrm{RD}$ are specified, respectively, as

$$
\begin{aligned}
& f(x)=2 \lambda^{2} x e^{-(\lambda x)^{2}}, \quad x \geq 0, \lambda>0, \\
& F(x)=1-e^{-(\lambda x)^{2}}, \quad x \geq 0, \lambda>0 .
\end{aligned}
$$

In the literature, there are many variants of the Rayleigh model which are used to provide a better fit of data. Voda [3] developed generalized Rayleigh and left-truncated model, Gomes et al. [4] suggested the Kumaraswamy generalized Rayleigh model, Merovci [5] suggested the transmuted Rayleigh model, Cordeiro et al. [6] suggested the beta generalized Rayleigh model. In [7], the inverse Rayleigh (IR) model was proposed to fit survival and reliability data. Voda 
[8] obtained estimates of the parameters of the IR model by ML estimation, Mukarjee and Maitim [9] discussed certain important statistical properties of the IR model, and Gharraph [10] developed expressions of descriptive statistics of the IR model. Moreover, Soliman et al. [11] and Gharraph [10] used maximum likelihood and Bayesian estimation methods to obtain estimates of parameters.

In recent times, the literature of probability models has been updated by inserting additional parameter(s) in the existing models using existing techniques available in literature or by proposing a new method to produce a better model as compared to the baseline model. Rehman and Sajjad [12] developed the IR model; Ahmad et al. [13] suggested transmuted the IR model, beta IR model [14], modified inverse Rayleigh model [15], generalized inverted scale family models [16], alpha power exponentiated inverse Rayleigh model [17], and alpha power Rayleigh distribution [18]. These newly proposed distributions were obtained and comparatively provided a better fit of the complex data.

The primary rationale behind producing modified Frechet-Rayleigh distribution (MFRD) is to overcome the inadequacy present in the Rayleigh model. The proposed distribution provides various shapes for hazard rate functions and shows more flexibility as compared to the Rayleigh distribution.

1.1. Research Problem. In practice, we might deal with data that exhibit monotonic or nonmonotonic hazard rate shapes. The available models may fail to be applied in such cases as in the case of life distributions. Therefore, researchers are trying to derive models that incorporate the deficiencies of these distributions. So, in this study, our aim is to propose a model that might efficiently fit such kind of data. This purpose is achieved by adding an extra parameter using generators or by the existing models; for detail, we refer to see [19]. Therefore, the modified Frechet technique is used to derive the proposed model.

1.2. Modified Frechet (MF) Technique. Alamgir et al. [20] suggested a novel technique, called modified Frechet technique (MFT) for developing new efficient probability models. The CDF and PDF of the newly developed technique are specified by the expressions given below:

$$
\begin{aligned}
G_{M F}(x) & =\frac{e^{-(F(x))^{\alpha}}-1}{\left(e^{-1}-1\right)}, \quad x>0 . \\
g_{M F}(x) & =\frac{\alpha f(x)(F(x))^{\alpha-1} e^{-(F(x))^{\alpha}}}{\left(1-e^{-1}\right)}, \quad x>0 .
\end{aligned}
$$

In equations (3) and (4), $F(x)$ and $f(x)$ denote the CDF and PDF of the input model, respectively. This technique is used to introduce modified Frechet-Rayleigh distribution (MFRD). The basic purpose of producing MFRD has a more flexible distribution to model life time data in comparison to other versions of Rayleigh distribution.

1.3. Modified Frechet-Rayleigh Distribution (MFRD). This section of the article introduces modified Frechet-Rayleigh distribution (MFRD).

The CDF of RD is specified by

$$
F(x)=1-e^{-(\lambda x)^{2}}, \quad x \geq 0, \lambda>0 .
$$

Definition 1. If $X \sim M F R D$, then its PDF is specified by $f_{\text {MFRD }}(x)=\frac{2 \alpha \lambda^{2} x e^{-(\lambda x)^{2}-\left(1-e^{-(\lambda x)^{2}}\right)^{\alpha}}\left\{1-e^{-(\lambda x)^{2}}\right\}^{(\alpha-1)}}{\left(1-e^{-1}\right)}, \quad x, \lambda, \alpha>0$.

The PDF and CDF are plotted in Figure 1.

The CDF of MFRD is specified by

$$
F_{\text {MFRD }}(x)=\frac{e^{-\left(1-e^{-(\lambda x)^{2}}\right)^{\alpha}}-1}{\left(e^{-1}-1\right)}, \quad \alpha, \lambda, x>0 .
$$

The hazard rate (HR) function of MFRD is defined by

$$
\begin{aligned}
h_{\text {MFRD }} & =\frac{\operatorname{pdf}}{\text { survivalfunction }}=\frac{2 \alpha \lambda^{2} x e^{-(\lambda x)^{2}-\left(1-e^{-(\lambda x)^{2}}\right)^{\alpha}}\left\{1-e^{-(\lambda x)^{2}}\right\}^{(\alpha-1)} /\left(1-e^{-1}\right)}{\left(e^{-1}-e^{-\left(1-e^{-(\lambda x)^{2}}\right)^{\alpha}}\right) /\left(e^{-1}-1\right)}, \\
h_{\text {AMKRD }}(x) & =\frac{2 \alpha \lambda^{2} x e^{-(\lambda x)^{2}-\left(1-e^{-(\lambda x)^{2}}\right)^{\alpha}}\left\{1-e^{-(\lambda x)^{2}}\right\}^{(\alpha-1)}}{\left(e^{-\left(1-e^{-(\lambda x)^{2}}\right)^{\alpha}}-e^{-1}\right)} .
\end{aligned}
$$



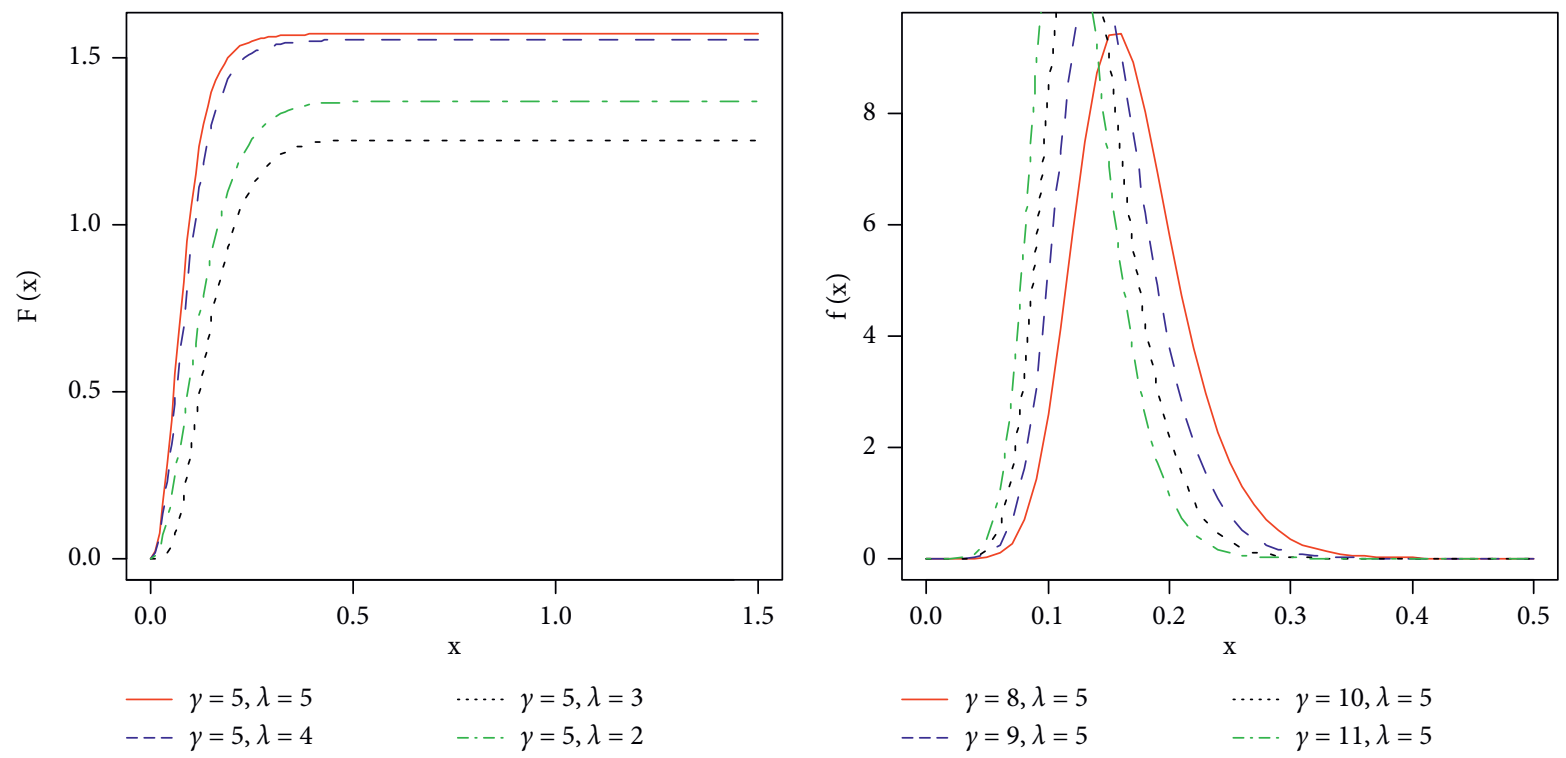

FIgURE 1: Graphs of PDF and CDF of MFRD.

The survival function (SF) of MFRD is specified by

$$
S_{\text {MFRD }}(x)=\frac{e^{-1}-e^{-\left(1-e^{-(\lambda x)^{2}}\right)^{\alpha}}}{\left(e^{-1}-1\right)} .
$$

Figure 2 exhibits graphs of HF and SF of MFRD.
Lemma 1. If $\alpha<1$ and $f(x)$ is nonincreasing function, then $f_{M F R D}(x)$ is also a nonincreasing function.

Proof. If $f(x)$ is differentiable and if $(d / d x) \log f(x)<0$ for $X$ in $(0, \infty)$, then $f(x)$ is nonincreasing and vice versa. Take $(d / d x) \log f_{\text {MFRD }}(x)<0$, that is,

$$
\begin{aligned}
& \frac{\mathrm{d}}{\mathrm{d} x} \log f_{\text {MFRD }}(x)=\frac{d}{d x} \log \left[\frac{2 \alpha \lambda^{2} x e^{-(\lambda x)^{2}-\left(1-e^{-(\lambda x)^{2}}\right)^{\alpha}\left\{1-e^{-(\lambda x)^{2}}\right\}^{(\alpha-1)}}}{\left(1-e^{-1}\right)}\right], \\
& \frac{\mathrm{d}}{\mathrm{d} x} \log f_{\text {MFRD }}(x)=\frac{d}{d x}\left[\log \left(2 \alpha \lambda^{2}\right)+\log x-(\lambda x)^{2}-\left(1-e^{-(\lambda x)^{2}}\right)^{\alpha}+(\alpha-1) \log \left\{1-e^{-(\lambda x)^{2}}\right\}-\log \left(1-e^{-1}\right)\right], \\
& \frac{\mathrm{d}}{\mathrm{d} x} \log f_{\text {MFRD }}(x)=\frac{1}{x}-2 \lambda^{2} x\left[1-\alpha e^{-(\lambda x)^{2}}\left(1-e^{-(\lambda x)^{2}}\right)^{(\alpha-1)}-\frac{(\alpha-1) e^{-(\lambda x)^{2}}}{\left(1-e^{-(\lambda x)^{2}}\right)}\right] .
\end{aligned}
$$

For nonnegative and less than 1 values of $\alpha$ and $\lambda>0$, (9) shows that $d / d x \log f_{\text {MFRD }}(x)<0$.

Hence, for $\alpha<1, \quad f_{\text {MFRD }}(x)$ is nonincreasing function.
Lemma 2. If $\alpha<1, f(x)$ is nonincreasing function, and $f(x)$ is log-convex, then $h_{M F R D}(x)$ is a nonincreasing function.

Proof. If $d^{2} / d x^{2} f(x)$ exists and $\left(d^{2} / d x^{2}\right) \log f(x)>0$, then $f(x)$ referred as log-convex.

By taking second differential of (10), we obtain

$$
\frac{\mathrm{d}^{2}}{\mathrm{~d} x^{2}} \log f_{\mathrm{MFRD}}(x)=\frac{d}{d x}\left[\frac{1}{x}-2 \lambda^{2} x\left[1-\alpha e^{-(\lambda x)^{2}}\left(1-e^{-(\lambda x)^{2}}\right)^{(\alpha-1)}-\frac{(\alpha-1) e^{-(\lambda x)^{2}}}{\left(1-e^{-(\lambda x)^{2}}\right)}\right]\right]
$$



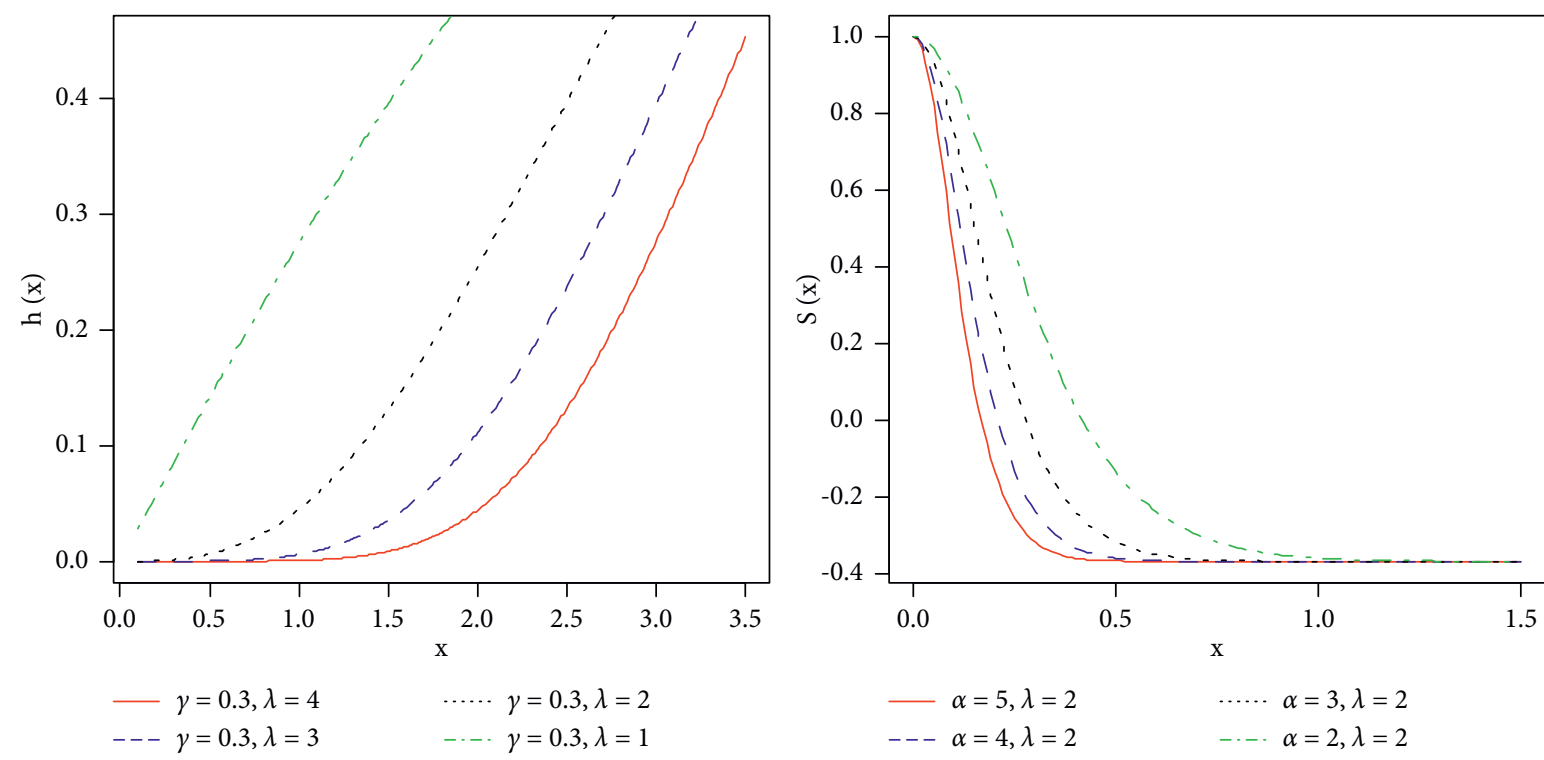

FIGURE 2: Graphs of HF and SF of MFRD.

$$
\begin{aligned}
\frac{\mathrm{d}^{2}}{\mathrm{~d} x^{2}} \log f_{\mathrm{MFRD}}(x)= & {\left[-\frac{1}{x^{2}}-2 \lambda^{2}-2 \alpha \lambda^{2} e^{-(\lambda x)^{2}}\left(1-e^{-(\lambda x)^{2}}\right)^{(\alpha-1)}\left[1-2 \lambda^{2} x^{2}+2 \lambda^{2}(\alpha-1) x^{2} e^{-(\lambda x)^{2}}\left(1-e^{-(\lambda x)^{2}}\right)^{-1}\right]\right.} \\
& \left.+2 \lambda^{2}(\alpha-1) e^{-(\lambda x)^{2}}\left(1-e^{-(\lambda x)^{2}}\right)^{-1}\left[1-2 \lambda^{2} x^{2}-2 \lambda^{2} x^{2} e^{-(\lambda x)^{2}}\left(1-e^{-(\lambda x)^{2}}\right)^{-1}\right]\right] .
\end{aligned}
$$

When $\alpha$ is nonnegative and less than 1 and $\lambda>0$, then $\left(\mathrm{d}^{2} / \mathrm{d} x^{2}\right) \log f_{\text {MFRD }}(x)>0$.

Therefore, for $0<\alpha<1, f_{\mathrm{MFRD}}(x)$ is log-convex [21].

1.4. Quantile Function (QF). If $X \sim \operatorname{MFRD}(\alpha, \lambda)$, then MFRD has the following QF: $F(x)=u$. This implies $x=F^{-1}(u)$, where the distribution of $u$ is uniform. The QF of MFRD is

$$
X_{p}=\frac{1}{\lambda}\left[-\log \left\{1-\left(-\log \left(u\left(e^{-1}-1\right)+1\right)\right)^{1 / \alpha}\right\}\right]^{1 / 2} .
$$

1.4.1. Median. By putting $u=1 / 2$ in (12), we get the median of MFRD as follows:

Median $=\left[\frac{1}{\lambda^{2}}\left\{-\log \left\{1-\left(-\log \left(\frac{e^{-1}+1}{2}\right)\right)^{1 / \alpha}\right\}\right\}\right]^{1 / 2}$.

1.4.2. Mode. To find mode of MFRD, solve the following for $x$ :

$$
\begin{gathered}
\frac{\mathrm{d}}{\mathrm{d} x} f_{\text {MFRD }}(x)=0 \Rightarrow \frac{\mathrm{d}}{\mathrm{d} x}\left(\frac{\left.2 \alpha \lambda^{2} x e^{-(\lambda x)^{2}-\left(1-e^{-(\lambda x)^{2}}\right)^{\alpha}\left\{1-e^{-(\lambda x)^{2}}\right\}^{(\alpha-1)}}\right)}{\left(1-e^{-1}\right)}\right) 0, \\
1-2 \lambda^{2} x^{2}\left[1+e^{-(\lambda x)^{2}}\left(1-e^{-(\lambda x)^{2}}\right)^{\alpha-1}\left\{\alpha-\frac{(\alpha-1) e^{-(\lambda x)^{2}}}{\left(1-e^{-(\lambda x)^{2}}\right)^{\alpha}}\right\}\right]=0 .
\end{gathered}
$$


Mode of the distribution satisfies (13).

1.4.3. $R^{\text {th }}$ Moment. Let $X \sim \operatorname{MFRD}(\alpha, \lambda)$; then, by definition,

$$
\begin{aligned}
\mu_{r}^{\prime} & =E\left(X^{r}\right) \\
& =\int_{0}^{\infty} x^{r} \frac{2 \alpha \lambda^{2} x e^{-(\lambda x)^{2}-\left(1-e^{-(\lambda x)^{2}}\right)^{\alpha}\left\{1-e^{-(\lambda x)^{2}}\right\}}}{\left(1-e^{-1}\right)} \mathrm{d} x .
\end{aligned}
$$

Put $e^{-(\lambda x)^{2}}=y$ in (15) and simplify; we obtain the expression below: $\mu_{r}^{\prime}=\mathrm{E}\left(X^{r}\right)=\alpha /\left(1-e^{-1}\right) \lambda^{r}(-1)^{r / 2} \int_{0}^{1}$ $(\log y)^{r / 2} e^{-(1-y)^{\alpha}}(1-y)^{(\alpha-1)} d y$.

Insert $(1-y)^{\alpha}=z$ in the above expression; after simplification, it will take the form given below:

$\mu_{r}^{\prime}=\frac{1}{\left(1-e^{-1}\right) \lambda^{r}}(-1)^{r / 2} \int_{0}^{1}\left\{\log \left(1-z^{1 / \alpha}\right)\right\}^{r / 2} e^{-z} \mathrm{~d} z$.

Insert series $e^{-z}=\sum_{k=0}^{\infty}(-z)^{k} / k$ ! and $\log \left(1-z^{1 / \alpha}\right)=$ $-\sum_{m=1}^{\infty}(-1)^{m}\left(-z^{1 / \alpha}\right)^{m} / m$ for $\left|z^{1 / \alpha}\right|<1$; from equation (16), we obtain the final expression as given below:

$$
\begin{aligned}
\mu_{r}^{\prime}= & \frac{1}{\left(1-e^{-1}\right) \lambda^{r}}(-1)^{r / 2} \int_{0}^{1} \\
& \left\{-\sum_{m=1}^{\infty} \frac{(-1)^{m}\left(-z^{1 / \alpha}\right)^{m}}{m}\right\}^{r / 2} \sum_{k=0}^{\infty} \frac{(-z)^{k}}{k !} \mathrm{d} z .
\end{aligned}
$$

The expression of $R^{\text {th }}$ moment is incomplete integral. It can be solved numerically using some numerical integration technique.

1.4.4. Moment Generating Function (MGF). Suppose $X$ : $\sim \operatorname{MFRD}(\alpha, \lambda)$; then, the MGF is specified by

$$
\begin{aligned}
M_{x}(t)= & E\left(e^{t x}\right)=\int_{0}^{\infty} e^{t x} \\
& \frac{2 \alpha \lambda^{2} x e^{-(\lambda x)^{2}-\left(1-e^{-(\lambda x)^{2}}\right)^{\alpha}\left\{1-e^{-(\lambda x)^{2}}\right\}^{(\alpha-1)}}}{\left(1-e^{-1}\right)} \mathrm{d} x .
\end{aligned}
$$

Insert $e^{t x}=\sum_{r=0}^{\infty} t^{r} x^{r} / r$ ! in (18), and after some calculation, we obtain

$$
\begin{aligned}
M_{x}(t)= & E\left(e^{t x}\right)=\sum_{r=0}^{\infty} \frac{t^{r}}{r !} \int_{0}^{\infty} x^{r} \\
& \frac{2 \alpha \lambda^{2} x e^{-(\lambda x)^{2}-\left(1-e^{-(\lambda x)^{2}}\right)^{\alpha}\left\{1-e^{-(\lambda x)^{2}}\right\}^{(\alpha-1)}}}{\left(1-e^{-1}\right)} \mathrm{d} x .
\end{aligned}
$$

Using result (15) in (19), we obtain

$$
\begin{aligned}
M_{x}(t)= & \frac{1}{\left(1-e^{-1}\right)} \sum_{r=0}^{\infty} \frac{t^{r}}{r ! \lambda^{r}}(-1)^{\frac{r}{2}} \int_{0}^{1} \\
& \left\{-\sum_{m=1}^{\infty} \frac{(-1)^{m}\left(-z^{1 / \alpha}\right)^{m}}{m}\right\}^{r / 2} \sum_{k=0}^{\infty} \frac{(-z)^{k}}{k !} \mathrm{d} z .
\end{aligned}
$$

The expression of MGF is an incomplete integral. It can be solved numerically by adopting any iterative method.

1.5. Order Statistics. Consider an order statistics $X_{1}, X_{2}, \ldots, X_{n}$ taken from MFRD. Then, the PDF of $i$ th order statistics $X_{i: n}$ is specified by

$$
f_{i: n}(x)=\frac{n !}{(i-1) !(n-i) !} f(x)[F(x)]^{i-1}[1-F(x)]^{(n-i)} \text {. }
$$

Insert (5) and (6) of MFRD in (21); we obtain $i^{\text {th }}$ order statistic is as follows:

$$
\begin{aligned}
& f_{i: n}(x)=\frac{n !}{(i-1) !(n-i) !}\left(\frac{2 \alpha \lambda^{2} x e^{-(\lambda x)^{2}-\left(1-e^{-(\lambda x)^{2}}\right)^{\alpha}}\left\{1-e^{-(\lambda x)^{2}}\right\}^{(\alpha-1)}}{\left(1-e^{-1}\right)}\right)\left[\frac{e^{-\left(1-e^{-(\lambda x)^{2}}\right)^{\alpha}}-1}{\left(e^{-1}-1\right)}\right]^{i-1}\left[1-\left(\frac{e^{-\left(1-e^{-(\lambda x)^{2}}\right)^{\alpha}-1}}{\left(e^{-1}-1\right)}\right)\right]^{(n-i)} \\
& f_{i: n}(x)=\frac{n !}{(i-1) !(n-i) !} \\
& \frac{1}{\left(1-e^{-1}\right)\left(e^{-1}-1\right)^{n-1}}\left[e^{-\left(1-e^{-(\lambda x)^{2}}\right)^{\alpha}}-1\right]^{(i-1)} 2 \alpha \lambda^{2} x e^{-(\lambda x)^{2}-\left(1-e^{-(\lambda x)^{2}}\right)^{\alpha}}\left\{1-e^{-(\lambda x)^{2}}\right\}^{(\alpha-1)}\left[e^{-1}-e^{-\left(1-e^{-(\lambda x)^{2}}\right)^{\alpha}}\right]^{(n-i)} .
\end{aligned}
$$


By putting $i=1$ in (22), we have expression for firstorder statistic as given below:

$$
f_{1: n}(x)=\frac{n}{\left(1-e^{-1}\right)\left(e^{-1}-1\right)^{n-1}} 2 \alpha \lambda^{2} x e^{-(\lambda x)^{2}-\left(1-e^{-(\lambda x)^{2}}\right)^{\alpha}}\left\{1-e^{-(\lambda x)^{2}}\right\}^{(\alpha-1)}\left[e^{-1}-e^{-\left(1-e^{-(\lambda x)^{2}}\right)^{\alpha}}\right]^{(n-1)} .
$$

By substituting $i=n$ in (22), the expression for the $\mathrm{n}^{\text {th }}$ order statistic will take the form given below:

$$
f_{n: n}(x)=\frac{n}{\left(1-e^{-1}\right)\left(e^{-1}-1\right)^{n-1}} 2 \alpha \lambda^{2} x e^{-(\lambda x)^{2}-\left(1-e^{-(\lambda x)^{2}}\right)^{\alpha}}\left\{1-e^{-(\lambda x)^{2}}\right\}^{(\alpha-1)}\left[e^{-\left(1-e^{-(\lambda x)^{2}}\right)^{\alpha}}-1\right]^{(n-1)} .
$$

For median's distribution insert $i=n / 2$ in (22), we have

$$
\begin{aligned}
f_{\frac{n}{2}: n}(x)= & \frac{n !}{((n / 2)-1) !(n-(n / 2)) !} \\
& \frac{1}{\left(1-e^{-1}\right)\left(e^{-1}-1\right)^{n-1}}\left[e^{-\left(1-e^{-(\lambda x)^{2}}\right)^{\alpha}}-1\right]^{((n / 2)-1)} 2 \alpha \lambda^{2} x e^{-(\lambda x)^{2}-\left(1-e^{-(\lambda x)^{2}}\right)^{\alpha}}\left\{1-e^{-(\lambda x)^{2}}\right\}^{(\alpha-1)}\left[e^{-1}-e^{-\left(1-e^{-(\lambda x)^{2}}\right)^{\alpha}}\right]^{(n-(n / 2))} .
\end{aligned}
$$

Lemma 3. The Shannon entropy (SE) of MFRD is given by

$$
\text { S.E } E_{x}=-\log \left[\frac{2^{1 / \alpha-1} \alpha^{2} \lambda}{\left(1-e^{-1}\right)^{2}} \int_{0}^{2}\left(-\sum_{k=1}^{\infty} \frac{(-1)^{k}\left(-2^{-1 / \alpha} z^{1 / \alpha}\right)^{k}}{k}\right)^{1 / 2} \sum_{m=0}^{\infty} \frac{(-z)^{m}}{m !}\left(2^{1 / \alpha}-z^{1 / \alpha}\right) z^{2-(2 / \alpha)} \mathrm{d} z\right] .
$$

Proof. The SE of MFRD is given as follows:

$$
\begin{aligned}
& \text { S. } E_{x}=E[-\log f(x)]=E\left[-\log \left\{\frac{2 \alpha \lambda^{2} x e^{-(\lambda x)^{2}-\left(1-e^{-(\lambda x)^{2}}\right)^{\alpha}\left\{1-e^{-(\lambda x)^{2}}\right\}^{(\alpha-1)}}}{\left(1-e^{-1}\right)}\right\}\right] \text {, } \\
& \text { S. } E_{x}=-\log \left[E\left(\frac{2 \alpha \lambda^{2} x e^{-(\lambda x)^{2}-\left(1-e^{-(\lambda x)^{2}}\right)^{\alpha}\left\{1-e^{-(\lambda x)^{2}}\right\}^{(\alpha-1)}}}{\left(1-e^{-1}\right)}\right)\right] \text {, } \\
& \text { S.E } x=-\log \left[\frac{4 \alpha^{2} \lambda^{4}}{\left(1-e^{-1}\right)^{2}} \int_{0}^{\infty} x \cdot x e^{-2(\lambda x)^{2}} e^{-2\left(1-e^{-(\lambda x)^{2}}\right)^{\alpha}}\left\{1-e^{-(\lambda x)^{2}}\right\}^{(2 \alpha-2)} \mathrm{d} x\right] \text {. }
\end{aligned}
$$


Substituting $1-e^{-(\lambda x)^{2}}=y$ in (27), the expression will be like

S.E $\mathrm{E}_{x}=-\log \left[\frac{2 \alpha^{2} \lambda}{\left(1-e^{-1}\right)^{2}} \int_{0}^{1}(-\log (1-y))^{1 / 2}(1-y) y^{2 \alpha-2} e^{-2 y^{\alpha}} \mathrm{d} y\right]$.
Putting $2 y^{\alpha}=z$ in (28), after simplification, it will take the following form:

$$
\text { S. } \mathrm{E}_{x}=-\log \left[\frac{2^{2 / \alpha-1} \alpha^{2} \lambda(-1)^{1 / 2}}{\left(1-e^{-1}\right)^{2}} \int_{0}^{2}\left[\log \left\{1-\left(\frac{1}{2} z\right)^{1 / \alpha}\right\}\right]^{1 / 2} e^{-z} z^{2-\frac{2}{\alpha}}\left(1-\left(\frac{z}{2}\right)^{1 / \alpha}\right) \mathrm{d} z\right] .
$$

Using series representation $e^{-z}=\sum_{m=0}^{\infty}(-z)^{m} / m$ ! and $\log \left(1-(z / 2)^{1 / \alpha}\right)=-\sum_{k=1}^{\infty}(-1)^{k}\left(-2^{-1 / \alpha} z^{1 / \alpha}\right)^{k} / k$ in (27), we get the final expression given below:

$$
S . E_{x}=-\log \left[\frac{2^{(1 / \alpha)-1} \alpha^{2} \lambda}{\left(1-e^{-1}\right)^{2}} \int_{0}^{2}\left(-\sum_{k=1}^{\infty} \frac{(-1)^{k}\left(-2^{-1 / \alpha} z^{1 / \alpha}\right)^{k}}{k}\right)^{1 / 2} \sum_{m=0}^{\infty} \frac{(-z)^{m}}{m !}\left(2^{1 / \alpha}-z^{1 / \alpha}\right) z^{2-(2 / \alpha)} \mathrm{d} z\right] .
$$

The final expression for SE is an incomplete integral. It can be solved numerically using numerical integration methods or some iterative procedures.

1.6. Mean Residual Life Function. Suppose $X$ follows MFRD. Then, $\mu(t)$ of MFRD has the expression given below:

$$
\mu(t)=\frac{1}{S(t)}\left(E(t)-\int_{0}^{t} x f(x) \mathrm{d} x\right)-t, \quad t \geq 0 .
$$

where

$$
\begin{aligned}
& \int_{0}^{t} x f(x) \mathrm{d} x \\
& =\int_{0}^{t} x \frac{2 \alpha \lambda^{2} x e^{-(\lambda x)^{2}-\left(1-e^{-(\lambda x)^{2}}\right)^{\alpha}\left\{1-e^{-(\lambda x)^{2}}\right\}^{(\alpha-1)}}}{\left(1-e^{-1}\right)} \mathrm{d} x .
\end{aligned}
$$

Putting, $1-e^{-(\lambda x)^{2}}=y$, the above expression will take the form given below:

$$
\int_{0}^{t} x f(x) \mathrm{d} x=\frac{\alpha(-1)^{1 / 2}}{\lambda\left(1-e^{-1}\right)} \int_{0}^{1-e^{-(\lambda t)^{2}}}(\log (1-y))^{1 / 2} e^{-y^{\alpha}} y^{\alpha-1} \mathrm{~d} y .
$$

Substitute $y^{\alpha}=z$ in (33); we obtain

$$
\int_{0}^{t} x f(x) \mathrm{d} x=\frac{(-1)^{1 / 2}}{\lambda\left(1-e^{-1}\right)} \int_{0}^{\left(1-e^{-(\lambda t)^{2}}\right)^{\alpha}}\left[\log \left(1-z^{1 / \alpha}\right)\right]^{1 / 2} e^{-z} \mathrm{~d} z
$$

Insert series $e^{-z}=\sum_{k=0}^{\infty}(-z)^{k} / k !$ and $\log \left(1-z^{1 / \alpha}\right)=$ $-\sum_{m=1}^{\infty}(-1)^{m}\left(-z^{1 / \alpha}\right)^{m} / m$ for $\left|z^{1 / \alpha}\right|<1$ in (31), we obtain

$$
\begin{gathered}
\int_{0}^{t} x f(x) \mathrm{d} x=\frac{(-1)^{1 / 2}}{\lambda\left(1-e^{-1}\right)} \int_{0}^{\left(1-e^{-(\lambda t)^{2}}\right)^{\alpha}}\left[-\sum_{m=1}^{\infty} \frac{(-1)^{m}\left(-z^{1 / \alpha}\right)^{m}}{m}\right]^{1 / 2} \sum_{k=0}^{\infty} \frac{(-z)^{k}}{k !} \mathrm{d} z, \\
E(t)=\int_{0}^{\infty} t f(t)=\int_{0}^{\infty} t \frac{2 \alpha \lambda^{2} t e^{-(\lambda t)^{2}-\left(1-e^{-(\lambda t)^{2}}\right)^{\alpha}\left\{1-e^{-(\lambda t)^{2}}\right\}^{(\alpha-1)}} \mathrm{d} t .}{\left(1-e^{-1}\right)}
\end{gathered}
$$

Substitute $1-e^{-(\lambda t)^{2}}=y$ in (33) and simplify; we have 


$$
E(t)=\frac{\alpha(-1)^{1 / 2}}{\lambda\left(1-e^{-1}\right)} \int_{0}^{1}(\log (1-y))^{1 / 2} e^{-y^{\alpha}} y^{\alpha-1} \mathrm{~d} y .
$$

Putting $y^{\alpha}=z$ in (37) and simplifying, we obtain

$$
E(t)=\frac{(-1)^{1 / 2}}{\lambda\left(1-e^{-1}\right)} \int_{0}^{1}\left[\log \left(1-z^{1 / \alpha}\right)\right]^{1 / 2} e^{-z} \mathrm{~d} z .
$$

Substitute series $e^{-z}=\sum_{k=0}^{\infty}(-z)^{k} / k !$ and $\log \left(1-z^{1 / \alpha}\right)=$ $-\sum_{m=1}^{\infty}(-1)^{m}\left(-z^{1 / \alpha}\right)^{m} / m$ for $\left|z^{1 / \alpha}\right|<1$ in (35), to have

$$
E(t)=\frac{(-1)^{1 / 2}}{\lambda\left(1-e^{-1}\right)} \int_{0}^{1}\left[-\sum_{m=1}^{\infty} \frac{(-1)^{m}\left(-z^{1 / \alpha}\right)^{m}}{m}\right]^{1 / 2} \sum_{k=0}^{\infty} \frac{(-z)^{k}}{k !} \mathrm{d} z .
$$

Put (8), (32), and (36) in (29); we obtain

$$
\begin{aligned}
& \mu(t)=\frac{(-1)^{1 / 2}\left(e^{-1}-1\right)}{\lambda\left(1-e^{-1}\right)\left(e^{-1}-e^{-\left(1-e^{-(\lambda x)^{2}}\right)^{\alpha}}\right)}\left[\left\{\int_{0}^{1}\left[-\sum_{m=1}^{\infty} \frac{(-1)^{m}\left(-z^{1 / \alpha}\right)^{m}}{m}\right]^{1 / 2} \sum_{k=0}^{\infty} \frac{(-z)^{k}}{k !} \mathrm{d} z\right\}\right. \\
& \left.-\left\{\int_{0}^{\left(1-e^{-(\lambda t)^{2}}\right)^{\alpha}}\left[-\sum_{m=1}^{\infty} \frac{(-1)^{m}\left(-z^{1 / \alpha}\right)^{m}}{m}\right]^{1 / 2} \sum_{k=0}^{\infty} \frac{(-z)^{k}}{k !} \mathrm{d} z\right\}\right]-t .
\end{aligned}
$$

The final expression of $\mu(t)$ is an incomplete integral. The expression in (40) can be solved numerically using any iterative method.

1.7. Stress-Strength Parameter (SSP). Let $X_{1}$ and $X_{2}$ be two random variables, which are independent and follow the same distribution such that $X_{1} \sim \operatorname{MFR} D\left(\alpha_{1}, \lambda\right)$ and
$X_{2} \sim \operatorname{MFRD}\left(\alpha_{2}, \lambda\right)$; then, the expression for the SSP is specified as

$$
R=\int_{-\infty}^{+\infty} f_{1}(x) F_{2}(x) \mathrm{d} x .
$$

Utilizing (5) and (6) in (41), then SSP is given as

$$
\begin{aligned}
& R=\int_{0}^{\infty}\left(\frac{2 \alpha_{1} \lambda^{2} x e^{-(\lambda x)^{2}-\left(1-e^{-(\lambda x)^{2}}\right)^{\alpha_{1}}}\left\{1-e^{-(\lambda x)^{2}}\right\}^{\left(\alpha_{1}-1\right)}}{\left(1-e^{-1}\right)}\right)\left(\frac{e^{-\left(1-e^{-(\lambda x)^{2}}\right)^{\alpha_{2}}}-1}{\left(e^{-1}-1\right)}\right) \mathrm{d} x, \\
& R=\frac{1}{\left(e^{-1}-1\right)\left(1-e^{-1}\right)} \int_{0}^{\infty} 2 \alpha_{1} \lambda^{2} x e^{-(\lambda x)^{2}} e^{-\left(1-e^{-(\lambda x)^{2}}\right)^{\alpha_{1}}}\left(1-e^{-(\lambda x)^{2}}\right)^{\alpha_{1}-1} e^{-\left(1-e^{-(\lambda x)^{2}}\right)^{\alpha_{2}}} \mathrm{~d} x-\frac{1}{\left(e^{-1}-1\right)} .
\end{aligned}
$$

Substitute $1-e^{-(\lambda x)^{2}}=y$ in (42) and simplify; we obtain $R=\frac{\alpha_{1}}{\left(e^{-1}-1\right)\left(1-e^{-1}\right)} \int_{0}^{1} e^{-y^{\alpha_{1}}} y^{\alpha_{1}-1} e^{-y^{\alpha_{2}}} d y-\frac{1}{\left(e^{-1}-1\right)}$.

Now, putting $y^{\alpha_{1}}=z$ in (43), after simplification, we get the expression given below:

$$
R=\frac{1}{\left(e^{-1}-1\right)\left(1-e^{-1}\right)} \int_{0}^{1} e^{-z} e^{-\frac{\alpha_{2}}{\alpha_{1}}} d z-\frac{1}{\left(e^{-1}-1\right)} .
$$

Put series $e^{-z}=\sum_{k=0}^{\infty}(-z)^{k} / k !$ and $e^{-z^{\alpha_{2} / \alpha_{1}}}=$ $\sum_{m=0}^{\infty}\left(-z^{\alpha_{2} / \alpha_{1}}\right)^{m} / m$ ! in (44); we get the following expression for SSP:

$$
\begin{aligned}
R= & \frac{1}{\left(e^{-1}-1\right)\left(1-e^{-1}\right)} \sum_{k=0}^{\infty} \sum_{m=0}^{\infty} \frac{(-1)^{k+m}}{k ! m !} \\
& \left(\frac{1}{k+c m+1}\right)-\frac{1}{\left(e^{-1}-1\right)}
\end{aligned}
$$

where $c=\alpha_{2} / \alpha_{1}$.

This is the required result.

\subsection{Parameters' Estimation}

1.8.1. MLE. The joint density function has the following expression: 


$$
\begin{aligned}
l(\alpha, \lambda)= & \left(\frac{2 \alpha \lambda^{2}}{1-e^{-1}}\right)^{n} \prod_{i=1}^{n} x_{i} e^{-\sum_{i=1}^{n}\left(\lambda x_{i}\right)^{2}} e^{-\sum_{i=1}^{n}\left(1-e^{-\left(\lambda x_{i}\right)^{2}}\right)^{\alpha}} \prod_{i=1}^{n} \\
& \left(1-e^{-\left(\lambda x_{i}\right)^{2}}\right)^{\alpha-1} .
\end{aligned}
$$

Taking natural logarithm of (46), we obtain

$\log l(\alpha, \lambda)=n \log \left(2 \lambda^{2} \alpha\right)-n \log \left(1-e^{-1}\right)+\sum_{i=1}^{n} \log x_{i}-\sum_{i=1}^{n}\left(\lambda x_{i}\right)^{2}-\sum_{i=1}^{n}\left(1-e^{-\left(\lambda x_{i}\right)^{2}}\right)^{\alpha}+(\alpha-1) \sum_{i=1}^{n} \log \left(1-e^{-\left(\lambda x_{i}\right)^{2}}\right)$

Differentiate (44) with respect to $\alpha$ and $\lambda$, respectively, to have the likelihood equations as

$$
\begin{aligned}
& \frac{\partial \log l(\alpha, \lambda)}{\partial \alpha}=\frac{n}{\alpha}-\sum_{i=1}^{n}\left(1-e^{-\left(\lambda x_{i}\right)^{2}}\right)^{\alpha} \log \left(1-e^{-\left(\lambda x_{i}\right)^{2}}\right)+\sum_{i=1}^{n} \log \left(1-e^{-\left(\lambda x_{i}\right)^{2}}\right)=0 \\
& \frac{\partial \log l(\alpha, \lambda)}{\partial \lambda}=\frac{2 n}{\lambda}-2 \lambda \sum_{i=1}^{n} x_{i}^{2}-2 \alpha \lambda \sum_{i=1}^{n} x_{i}^{2} e^{-\left(\lambda x_{i}\right)^{2}}\left(1-e^{-\left(\lambda x_{i}\right)^{2}}\right)^{(\alpha-1)}+2 \lambda(\alpha-1) \sum_{i=1}^{n} \frac{x_{i}^{2} e^{-\left(\lambda x_{i}\right)^{2}}}{\left(1-e^{-\left(\lambda x_{i}\right)^{2}}\right)^{(\alpha-1)}}=0 .
\end{aligned}
$$

The solution of above equations is obtained using the Newton-Raphson method. For large sample size, the ML Estimators are normally distributed, that is, $\sqrt{n}(\widehat{\alpha}-\alpha, \widehat{\lambda}-\lambda) \sim N_{2}(0, \Sigma)$, where $\Sigma$ denotes variance covariance matrix acquired by inverting Fisher information matrix $F$ as follows:

$$
F=\left(\begin{array}{ll}
\frac{\partial^{2} \log l}{\partial \alpha^{2}} & \frac{\partial^{2} \log l}{\partial \alpha \partial \lambda} \\
\frac{\partial^{2} \log l}{\partial \alpha \partial \lambda} & \frac{\partial^{2} \log l}{\partial \lambda^{2}}
\end{array}\right)
$$

Again differentiating (45) and (46) with respect to $\alpha$ and $\lambda$, respectively, we obtain

$$
\begin{aligned}
\frac{\partial^{2} \log l}{\partial \alpha^{2}}= & \frac{-n}{\alpha^{2}}-\sum_{i=1}^{n}\left(1-e^{-\left(\lambda x_{i}\right)^{2}}\right)^{\alpha}\left(\log \left(1-e^{-\left(\lambda x_{i}\right)^{2}}\right)\right)^{2} \\
\frac{\partial^{2} \log l}{\partial \lambda^{2}}= & {\left[\left\{-\frac{2 n}{\lambda^{2}}+2 \sum_{i=1}^{n} x_{i}^{2}+2 \alpha \sum_{i=1}^{n} x_{i}{ }^{2} e^{-\left(\lambda x_{i}\right)^{2}}\left(1-e^{-\left(\lambda x_{i}\right)^{2}}\right)^{(\alpha-1)}-4 \alpha \lambda^{2} \sum_{i=1}^{n} x_{i}^{4} e^{-\left(\lambda x_{i}\right)^{2}}\left(1-e^{-\left(\lambda x_{i}\right)^{2}}\right)^{(\alpha-1)}\right.\right.} \\
& \left.+4 \alpha(\alpha-1) \lambda^{2} \sum_{i=1}^{n} x_{i}^{4} e^{-2\left(\lambda x_{i}\right)^{2}}\left(1-e^{-\left(\lambda x_{i}\right)^{2}}\right)^{(\alpha-2)}\right\}+\left\{2(\alpha-1) \sum_{i=1}^{n} x_{i}{ }^{2} e^{-\left(\lambda x_{i}\right)^{2}}\left(1-e^{-\left(\lambda x_{i}\right)^{2}}\right)^{(-\alpha+1)}\right. \\
& \left.\left.-4 \lambda^{2}(\alpha-1) \sum_{i=1}^{n} x_{i}{ }^{4} e^{-\left(\lambda x_{i}\right)^{2}}\left(1-e^{-\left(\lambda x_{i}\right)^{2}}\right)^{(-\alpha+1)}-4(\alpha-1)^{2} \lambda^{2} \sum_{i=1}^{n} x_{i}^{4} e^{-2\left(\lambda x_{i}\right)^{2}}\left(1-e^{-\left(\lambda x_{i}\right)^{2}}\right)^{-\alpha}\right\}\right]
\end{aligned}
$$


TABLE 1: Simulation results.

\begin{tabular}{|c|c|c|c|c|c|}
\hline Parameter & $\mathrm{N}$ & $\operatorname{MSE}(\widehat{\alpha})$ & $\operatorname{MSE}(\widehat{\lambda})$ & $\operatorname{Bias}(\widehat{\alpha})$ & $\operatorname{Bias}(\widehat{\lambda})$ \\
\hline \multirow{3}{*}{$\begin{array}{l}\alpha=0.3635288 \\
\lambda=0.4765585\end{array}$} & 50 & 0.002578026 & 0.2981227 & 0.01997809 & -0.2981874 \\
\hline & 100 & 0.001316036 & 0.1959489 & 0.003959582 & -0.195388 \\
\hline & 200 & 0.000679110 & 0.05703854 & 0.003634784 & -0.05379642 \\
\hline \multirow{3}{*}{$\begin{array}{l}\alpha=0.3735288 \\
\lambda=0.3735288\end{array}$} & 50 & 0.0029977 & 0.2109166 & 0.008424304 & -0.2712479 \\
\hline & 100 & 0.001779635 & 0.1441912 & 0.004146879 & -0.1916479 \\
\hline & 200 & 0.000819825 & 0.09560007 & 0.003931851 & -0.1200957 \\
\hline \multirow{3}{*}{$\begin{array}{l}\alpha=0.4048975 \\
\lambda=0.3565585\end{array}$} & 50 & 0.004289024 & 0.2029563 & 0.02249881 & -0.2622389 \\
\hline & 100 & 0.00210558 & 0.1442519 & 0.01043012 & -0.1901263 \\
\hline & 200 & 0.000884641 & 0.07766141 & 0.004018672 & -0.10063 \\
\hline \multirow{3}{*}{$\begin{array}{l}\alpha=0.4335288 \\
\lambda=0.3865585\end{array}$} & 50 & 0.005570932 & 0.2722905 & 0.01798329 & -0.3314039 \\
\hline & 100 & 0.001580699 & 0.1930487 & 0.006078905 & -0.236486 \\
\hline & 200 & 0.001143901 & 0.09653606 & 0.001407309 & -0.1170932 \\
\hline \multirow{3}{*}{$\begin{array}{l}\alpha=0.4535288 \\
\lambda=0.4065585\end{array}$} & 50 & 0.005564972 & 0.2572082 & 0.0184824 & -0.3021972 \\
\hline & 100 & 0.002564085 & 0.2113778 & 0.01034615 & -0.2478211 \\
\hline & 200 & 0.001494582 & 0.1127926 & 0.007373421 & -0.1322202 \\
\hline \multirow{3}{*}{$\begin{array}{l}\alpha=0.4648975 \\
\lambda=0.3565585\end{array}$} & 50 & 0.004006028 & 0.2210296 & 0.02249881 & -0.2946936 \\
\hline & 100 & 0.002153703 & 0.1737456 & 0.00791557 & -0.231912 \\
\hline & 200 & 0.001177539 & 0.0773178 & 0.002197945 & -0.1055793 \\
\hline \multirow{3}{*}{$\begin{array}{l}\alpha=0.4735288 \\
\lambda=0.3865585\end{array}$} & 50 & 0.005596782 & 0.2460749 & 0.02127052 & -0.3002337 \\
\hline & 100 & 0.00203143 & 0.1974326 & 0.00842539 & -0.245515 \\
\hline & 200 & 0.001512619 & 0.07987172 & 0.005135786 & -0.09911058 \\
\hline
\end{tabular}

TABLE 2: Goodness of fit result for dataset 1.

\begin{tabular}{lcccccccc}
\hline Model & \multicolumn{2}{c}{ MLE } & AIC & CAIC & BIC & HQIC & K-S & $p$-value \\
\hline MFRD & 0.9691 & 0.3959 & 208.0488 & 208.2227 & 212.6022 & 209.8615 & 0.1194 & 0.2561 \\
RD & -0.4542 & & 212.2285 & 212.2856 & 214.5051 & 213.1348 & 0.1404 & 0.1172 \\
MIRD & 1.2641 & -0.0484 & 247.3044 & 247.4783 & 251.8578 & 249.1171 & 0.2347 & 0.0007 \\
GIRD & 0.3924 & 2.2022 & 290.9948 & 291.1687 & 295.5482 & 292.8075 & 0.7977 & $2.2 e-16$ \\
TIRD & 0.3558 & -0.9404 & 294.6214 & 294.7953 & 299.1747 & 296.4341 & 0.3506 & $4.106 e-08$ \\
EIRD & 0.2285 & 2.0379 & 341.9955 & 342.1694 & 346.5488 & 343.8082 & 0.4750 & $1.554 e-14$ \\
\hline
\end{tabular}

Again differentiating (45) w.r.t $\lambda$, we obtain

$$
\begin{gathered}
\frac{\partial^{2} \log l}{\partial \alpha \partial \lambda}=-2 \lambda^{2} \alpha \sum_{i=1}^{n} x_{i} e^{-\left(\lambda x_{i}\right)^{2}}\left(1-e^{-\left(\lambda x_{i}\right)^{2}}\right)^{(\alpha-1)} \log \\
\left(1-e^{-\left(\lambda x_{i}\right)^{2}}\right)+2 \lambda \sum_{i=1}^{n} \frac{x_{i} e^{-\left(\lambda x_{i}\right)^{2}}}{\left(1-e^{-\left(\lambda x_{i}\right)^{2}}\right)} .
\end{gathered}
$$

Asymptotic $(1-\zeta) 100 \%$ confidence intervals for parameters of MFRD are obtained by $\widehat{\alpha} \pm Z_{\zeta / 2} \sqrt{\Sigma_{11}}$ and $\hat{\lambda} \pm Z_{\zeta / 2} \sqrt{\Sigma_{22}}$, where $Z_{\zeta}$ denote the upper $\zeta^{\text {th }}$ percentile of the standard normal distribution.

1.9. Simulation Study. To assess the consistency of MLE, the MSE and bias are measured based on $W=1000$ simulations. Samples having sizes 50, 100, and 200 are generated from MFRD using the following expression of the quantile function:

$$
X_{p}=\frac{1}{\lambda}\left[-\log \left\{1-\left(-\log \left(u\left(e^{-1}-1\right)+1\right)\right)^{1 / \alpha}\right\}\right]^{1 / 2},
$$

where $U$ follows uniform distribution over $[0,1]$. Bias and MSE of MFRD are computed by the following expressions:

$$
\begin{aligned}
\text { Bias } & =\frac{1}{W} \sum_{l=1}^{w}\left(\widehat{b}_{i}-b\right), \\
\text { MSE } & =\frac{1}{W} \sum_{l=1}^{w}\left(\widehat{b}_{i}-b\right)^{2},
\end{aligned}
$$

where $b=(\alpha, \lambda)$. Simulation results were obtained for various values of $\alpha$ and $\lambda$. The results are shown in Table 1 . The consistency pattern can easily be observed as estimates' approach to their true values by increasing the sample size. Furthermore, the values of error measures of the parameter estimates' decrease for all combinations of parameter values. Hence, it can be concluded that MLE procedure produces consistent estimates in estimating the parameters of MFRD. The same consistency of parameters has been observed for all choices of parameter values. It is also evident from Table 1 that bias for the estimators are decreasing with increase in the sample sizes and parameter $\alpha$. Therefore, we conclude that these estimators are asymptotically unbiased.

\section{Applications}

To assess the performance of the MFRD, two practical datasets are used and the results are compared using some standard model selection criteria's. The proposed model is 
TABLE 3: Goodness-of-fit result for dataset 2.

\begin{tabular}{lcccccccc}
\hline Model & \multicolumn{2}{c}{ MLE } & AIC & CAIC & BIC & HQIC & K-S & $p$-value \\
\hline MFRD & 1.1859 & -0.0603 & 394.472 & 394.686 & 398.627 & 396.094 & 0.160 & 0.095 \\
RD & 0.0638 & & 397.421 & 397.491 & 399.499 & 398.232 & 0.172 & 0.060 \\
MIRD & 8.4846 & 12.2370 & 416.948 & 417.162 & 421.103 & 418.570 & 0.253 & 0.001 \\
GRD & 1.0295 & 0.0643 & 399.393 & 399.607 & 403.548 & 401.015 & 0.177 & 0.049 \\
\hline
\end{tabular}
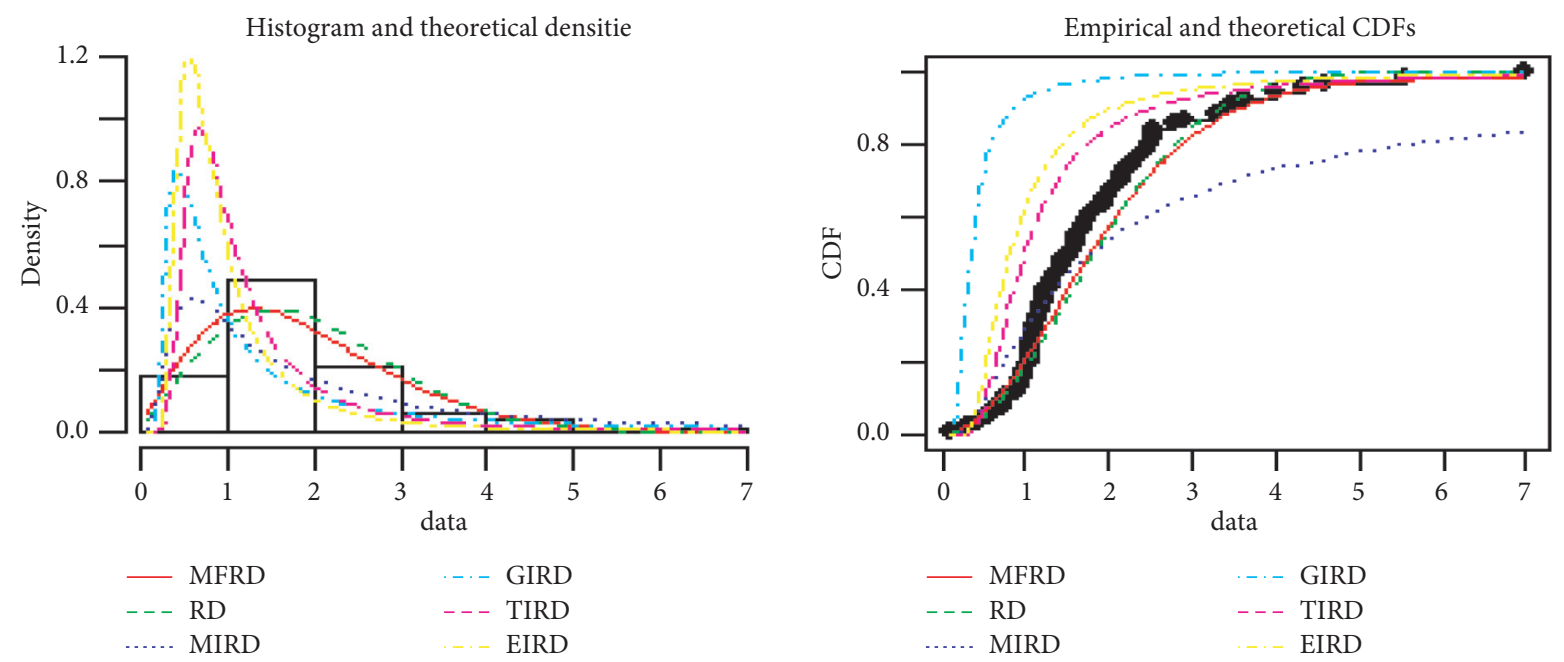

FIgURE 3: Comparison of MFRD with other competitive models for dataset 1.
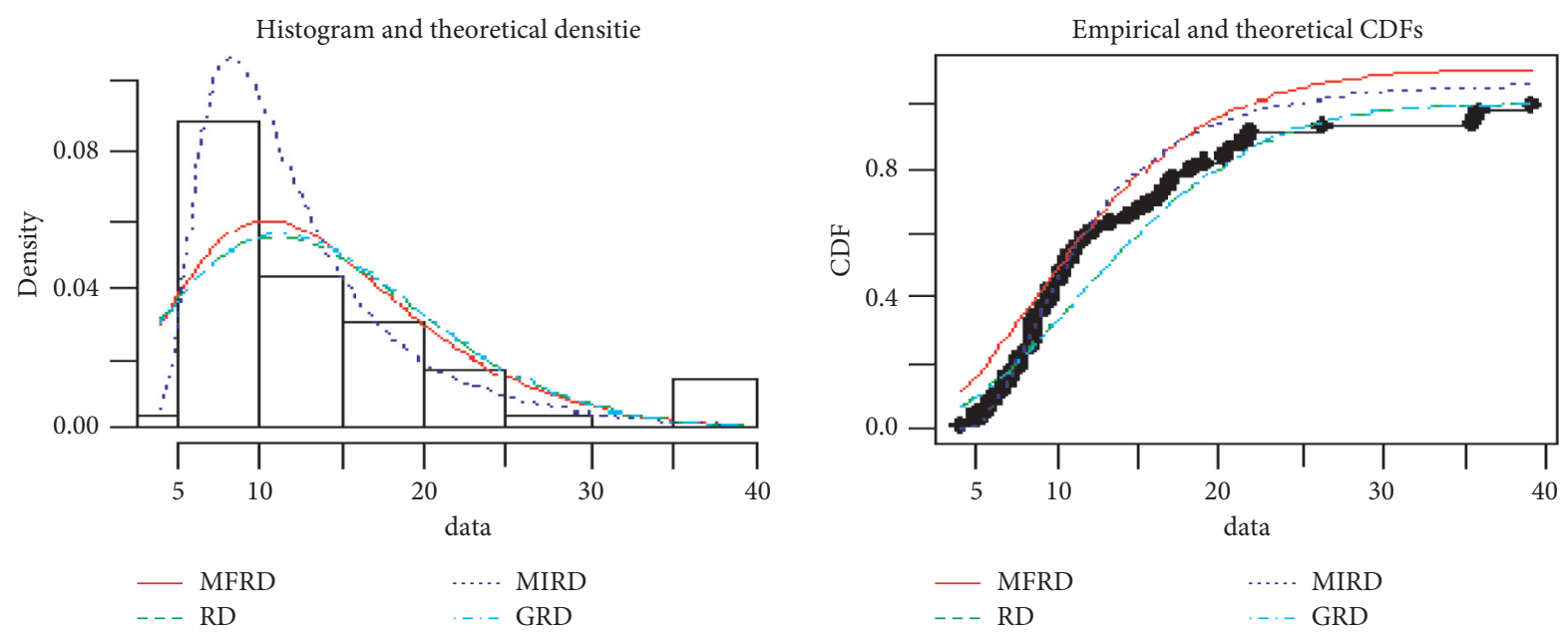

FIGURE 4: Comparison of MFRD with other competitive models for dataset 2. 

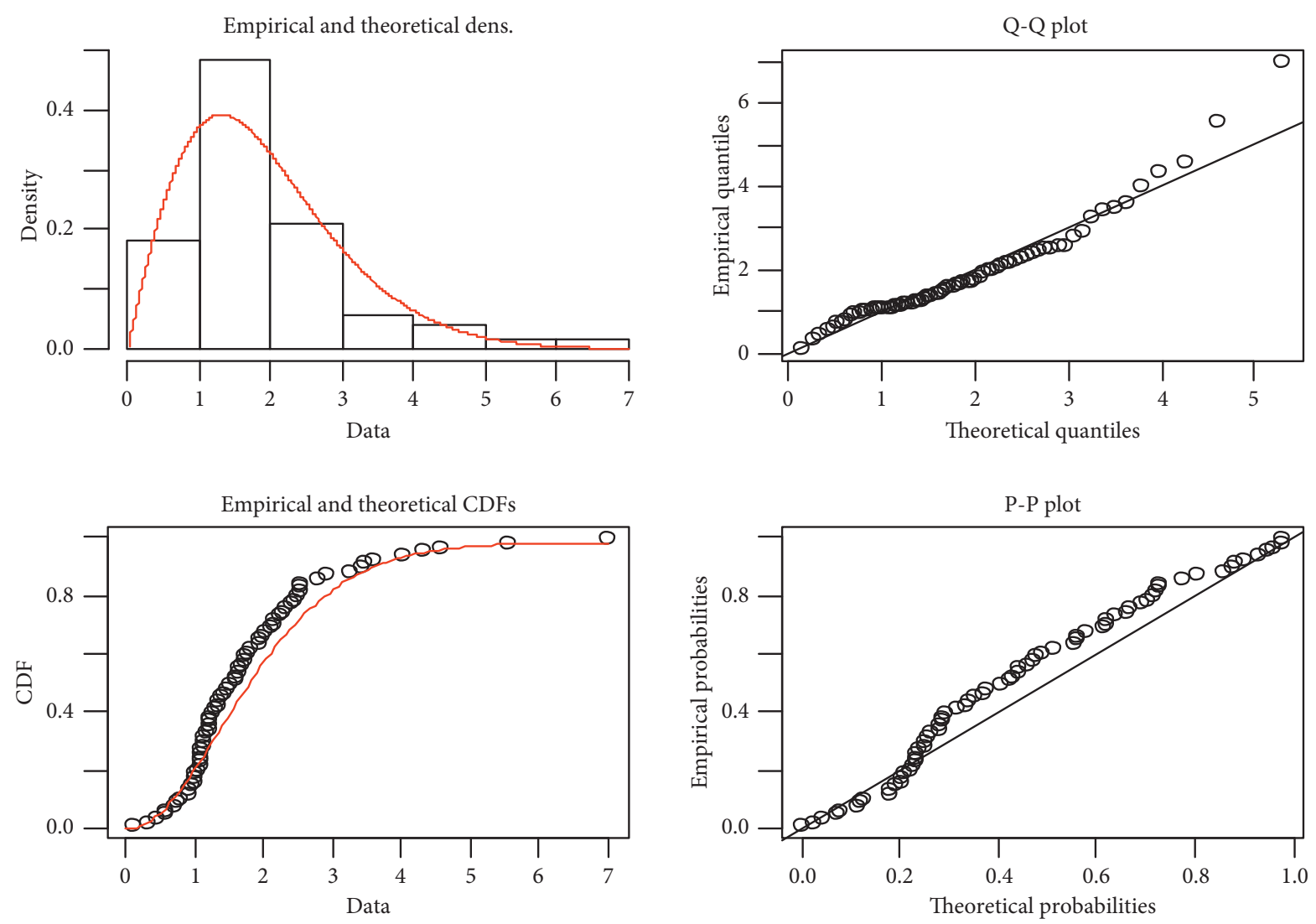

Figure 5: Plots of MFRD for dataset 1.
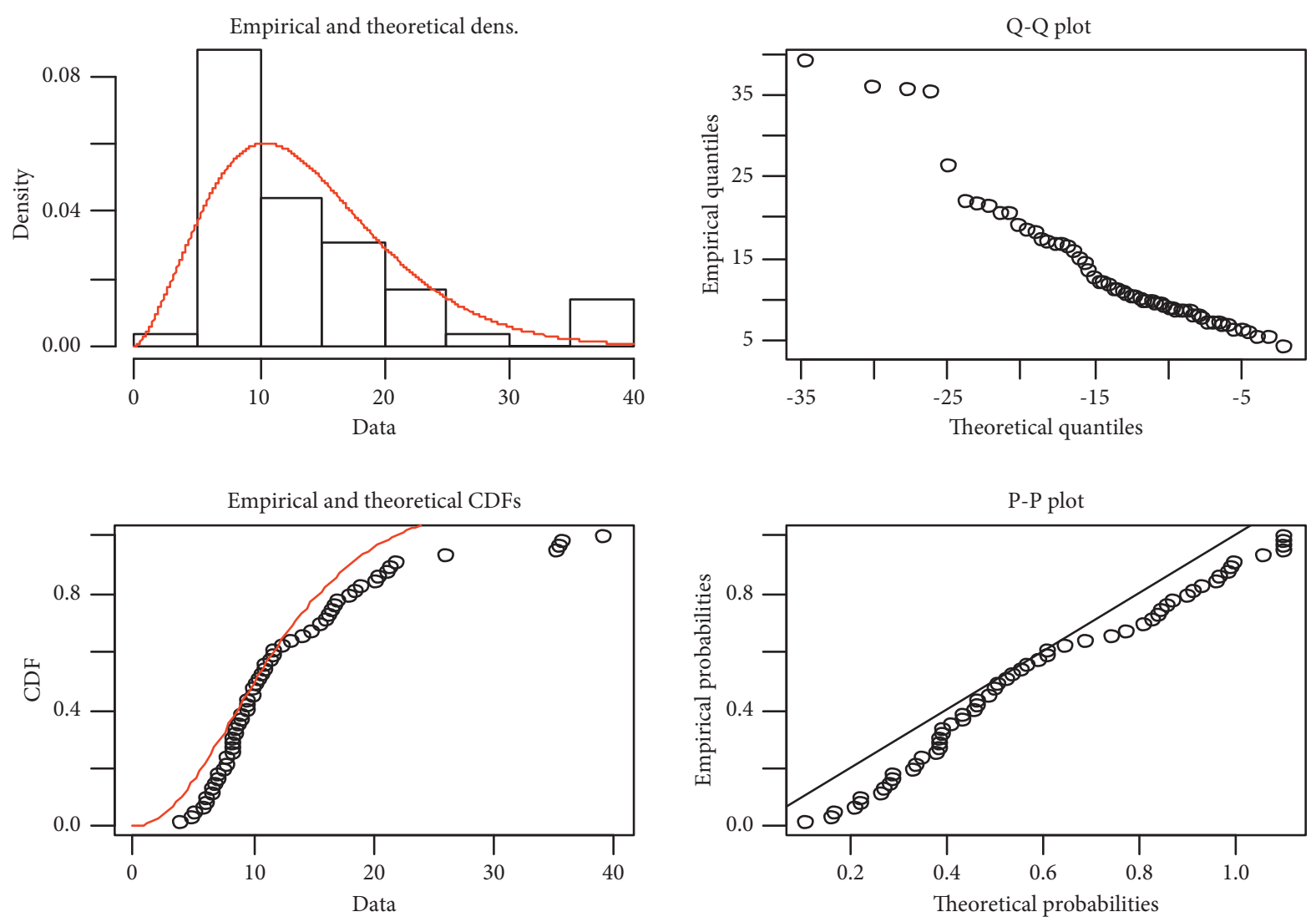

Figure 6: Plots of MFRD for dataset 2. 
compared with Rayleigh distribution (RD) [22], modified inverse Rayleigh distribution (MIRD) [15], generalized inverse Rayleigh distribution (GIRD) [16], transmuted inverse Rayleigh distribution (TIRD) (Ahmad et al., 2014), exponentiated inverse Rayleigh distribution (EIRD) [12], and generalized Rayleigh distribution (GRD) [23].

2.1. Dataset 1. Dataset 1 was taken from Bjerkedal [24]. The data points are given below: $0.1,0.33,1.08,1.08,1.08,0.44$, $0.56,0.59,0.72,0.74,0.77,2.54,2.78,2.93,3.27,3.42,0.92$, $0.93,0.96,1,1,1.02,1.05,1.07,07,1.09,1.12,1.13,1.15,1.36$, $1.39,1.44,1.83,1.95,1.96,1.97,2.02,1.16,1.2,1.21,1.22$, $1.22,1.24,1.3,1.34,2.13,1.46,1.53,1.59,1.6,1.63,1.63,1.68$, $1.71,1.72,1.76,2.15,2.16,2.22,2.3,2.31,2.4,2.45,2.51$, $2.53,2.54,3.47,3.61,4.02,4.32,4.58,5.55$.

2.2. Dataset 2. Dataset 2 is taken from Nassar and Nada [25]. The data points are as follows: $5.9,20.4,14.9,16.2,17.2,7.8$, $6.1,9.2,10.2,9.6,13.3,8.5,21.6,18.5,5.1,6.7,17,8.6,9.7$, $39.2,35.7,15.7,9.7,10,4.1,36,8.5,8,9.2,26.2,21.9,16.7$, $21.3,35.4,14.3,8.5,10.6,19.1,20.5,7.1,7.7,18.1,16.5,11.9,7$, 8.6, 12.5, 10.3, 11.2, 6.1, 8.4, 11, 11.6, 11.9, 5.2, 6.8, 8.9, 7.1, 10.8 .

$P$-value and some well-known standard model selection criteria's are used for comparison purposes. The results are displayed in Tables 2 and 3 of dataset 1 and 2, respectively. Comparative analysis of different distributions based on two data points are shown in Figures 3 and 4, respectively.

The results of Tables 2 and 3 indicate that MFRD provides improved result in comparison to other versions of the Rayleigh model on the basis of well-known model selection criteria. Figure 5 and 6 provide PP-plots, QQ-plots, plots of $\mathrm{PDF}$, and CDF for the two datasets.

\section{Conclusion}

In this article, we suggested a new lifetime model referred to as modified Frechet-Rayleigh distribution (MFRD) using the modified Frechet technique. Several statistical properties of the MFRD were obtained such as moments, MGF, and median in closed form, stress-strength parameter, Quantile function, mean residuals' life function, order statistics, and the expression for entropies. The parameters of the proposed model were estimated using the MLE approach. The simulation results revealed that the estimates of the parameters of MFRD are consistent and asymptotically unbiased. The performance of MFRD was evaluated on the basis of real datasets. The results indicated better performance of MFRD as compared to various versions of Rayleigh distribution.

\section{Data Availability}

The data used to support the findings of this study are available from the corresponding author upon request.

\section{Conflicts of Interest}

The authors declare that they have no conflicts of interest to report regarding the present study.

\section{Authors' Contributions}

All authors have equally contributed to the research design, data analysis research summary and recommendation, written manuscript, and coordinated for submission of this paper.

\section{References}

[1] S. Aja-Fernández, C. Alberola-López, and C.-F. Westin, "Noise and signal estimation in magnitude MRI and Rician distributed images: a LMMSE approach," IEEE Transactions on Image Processing, vol. 17, no. 8, pp. 1383-1398, 2008.

[2] A. S. Akhter and A. S. Hirai, "Estimation of the scale parameter from the Rayleigh distribution from type II singly and doubly censored data," Pakistan Journal of Statistics and Operation Research, vol. 5, no. 1, pp. 31-45, 2009.

[3] V. G. Voda, "Note on the truncated Rayleigh variate," Revista Colombiana de Matemáticas, vol. 9, no. 1, pp. 1-7, 1975.

[4] A. E. Gomes, C. Q. da-Silva, G. M. Cordeiro, and E. M. M. Ortega, "A new lifetime model: the Kumaraswamy generalized Rayleigh distribution," Journal of Statistical Computation and Simulation, vol. 84, no. 2, pp. 290-309, 2014.

[5] F. Merovci, "Transmuted Rayleigh distribution," Austrian Journal of statistics, vol. 42, no. 1, pp. 21-31, 2013.

[6] G. M. Cordeiro, C. T. Cristino, E. M. Hashimoto, and E. M. M. Ortega, "The beta generalized Rayleigh distribution with applications to lifetime data," Statistical Papers, vol. 54, no. 1, pp. 133-161, 2013.

[7] V. N. Trayer, "Inverse Rayleigh (IR) model," in Proceedings of the Academy of Science, Doklady Akad, Nauk Belarus, U.S.S.R, 1964.

[8] V. G. Voda, "On the inverse Rayleigh distributed random variable," Rep. Statis. App. Res. JUSE, vol. 19, no. 1, pp. 13-21, 1972.

[9] S. P. Mukherjee and S. S. Maiti, "A percentile estimator of the inverse Rayleigh parameter," IAPqR Transactions, vol. 21, pp. 63-66, 1996.

[10] M. K. Gharraph, "Comparison of estimators of location measures of an inverse Rayleigh distribution," The Egyptian Statistical Journal, vol. 37, no. 2, pp. 295-309, 1993.

[11] A. Soliman, E. A. Amin, and A. A. Abd-El Aziz, "Estimation and prediction from inverse Rayleigh distribution based on lower record values," Applied Mathematical Sciences, vol. 4, no. 62, pp. 3057-3066, 2010.

[12] S. Rehman, I. Dar, and Sajjad, "Bayesian analysis of exponentiated Inverse Rayleigh distribution under different Priors," M.Phil. thesis, 2015.

[13] A. Ahmad, S. P. Ahmad, and A. Ahmed, "Transmuted inverse Rayleigh distribution: a generalization of the inverse Rayleigh distribution," Mathematical Theory and Modeling, vol. 4, no. 7, pp. 90-98, 2014.

[14] J. Leao, H. Saulo, M. Bourguignon, R. Cintra, L. Rêgo, and G. Cordeiro, "On some properties of the beta inverse Rayleigh 
distribution," Chilean Journal of Statistics, vol. 4, no. 2, pp. 111-131, 2013.

[15] M. ShuaibKhan, "Modified inverse Rayleigh distribution," International Journal of Computer Application, vol. 87, no. 13, pp. 28-33, 2014.

[16] K. G. Potdar and D. T. Shirke, "Inference for the parameters of generalized inverted family of distributions," ProbStat Forum, vol. 6, pp. 18-28, 2013.

[17] M. Ali, A. Khalil, M. Ijaz, and N. Saeed, "Alpha-Power Exponentiated Inverse Rayleigh distribution and its applications to real and simulated data," PLoS One, vol. 16, no. 1, Article ID e0245253, 2021.

[18] A. S. Malik and S. P. Ahmad, "Alpha power Rayleigh distribution and its application to life time data," International Journal of Enhanced Research in Management \& Computer Applications, vol. 6, no. 11, pp. 212-219, 2017.

[19] S. Dey, A. Alzaatreh, C. Zhang, and D. Kumar, "A new extension of generalized exponential distribution with application to ozone data," Ozone: Science \& Engineering, vol. 39, no. 4, pp. 273-285, 2017.

[20] A. Khalil, A. A. H. Ahmadini, M. Ali, W. K. Mashwani, S. S. Alshqaq, and Z. Salleh, "A novel method for developing efficient probability distributions with applications to engineering and life science data," Journal of Mathematics, vol. 2021, Article ID 4479270, 13 pages, 2021.

[21] S. Boyd, S. P. Boyd, and L. Vandenberghe, Convex Optimization, Cambridge University Press, Cambridge, UK, 2004.

[22] L. Rayleigh, "XII. On the resultant of a large number of vibrations of the same pitch and of arbitrary phase," The London, Edinburgh, and Dublin Philosophical Magazine and Journal of Science, vol. 10, no. 60, pp. 73-78, 1880.

[23] M. Z. Raqab and M. T. Madi, "Generalized Rayleigh distribution," International Encyclopedia of Statistical Science, vol. 5, no. 2, pp. 599-603, 2011.

[24] T. Bjerkedal, "Acquisition of resistance in Guinea pigs infected with different doses of virulent tubercle Bacilli1," American Journal of Epidemiology, vol. 72, no. 1, pp. 130-148, 1960.

[25] M. M. Nassar and N. K. Nada, "The beta generalized Pareto distribution," Journal of Statistics: Advances in Theory and Applications, vol. 6, no. 1/2, pp. 1-17, 2011. 